\title{
A Review on Neural Dynamics for Robot Autonomy
}

\author{
Dechao Chen ${ }^{1}$, Shuai $\mathrm{Li}^{* 2}$, Qing $\mathrm{Wu}^{1}$ \\ ${ }^{1}$ School of Computer Science and Technology, Hangzhou Dianzi University, Hangzhou, P.R. China \\ ${ }^{2}$ Department of Computing, The Hong Kong Polytechnic University, Hung Hom, Kowloon, Hong Kong, China
}

Received: June 21, 2018

DOI: $10.5430 /$ ijrc.v1n $1 \mathrm{p} 20$

\author{
Accepted: July 16, 2018 \\ Online Published: August 2, 2018 \\ URL: https://doi.org/10.5430/ijrc.v1n1p20
}

\begin{abstract}
Exploiting neural networks to solve control problems of robots is becoming commonly and effectively in academia and engineering. Due to the remarkable features like distributed storage, parallelism, easy implementation by hardware, adaptive self-learning capability, and free of off-line training, the solutions of neural networks break the bottlenecks of serial-processing strategies and methods, and serve as significant alternatives for robotic engineers and researchers. Especially, various types and branches of recurrent neural networks (RNNs) have been sequentially developed since the seminal works by Hopfield and Tank. Successively, many classes and branches of RNNs such as primal-dual neural networks (PDNNs), zeroing neural networks (ZNNs) and gradient neural networks (GNNs) are proposed, investigated, developed and applied to the robot autonomy. The objective of this paper is to present a comprehensive review of the research on neural networks (especially RNNs) for control problems solving of different kinds of robots. Specifically, the state-of-the-art research of RNNs, PDNNs, ZNNs and GNNs in different robot control problems solving are detailedly revisited and reported. The readers can readily find many effective and valuable solutions on the basis of neural networks for the robot autonomy in this paper.
\end{abstract}

Key Words: Neural dynamics, Robot autonomy, Recurrent neural networks, Zeroing neural networks, Primal-dual neural networks, Gradient neural networks

\section{INTRODUCTION}

Neural networks (or termed artificial neural networks) are simple systems that are composed of many connected neurons to simulate the structure of human brain. ${ }^{[1-5]}$ Neural networks usually possess the characteristics of adaptivity, nonlinearity, parallelism and distributed storage, which can be used to solve the complicated problems that can not be solved by other approaches. ${ }^{[6-9]}$ Specifically, the applications of neural networks including (but not limited to) the pattern classification, ${ }^{[10,11]}$ deep learning, ${ }^{[12,13]}$ approximation and prediction, ${ }^{[14,15]}$ image processing, ${ }^{[16,17]}$ machine learning, ${ }^{[18,19]}$ optimization and computation, ${ }^{[20,21]}$ complex system control ${ }^{[22-24]}$ (including the robot system control). ${ }^{[25,26]}$ Due to the extensive and significant applications of neu- ral networks, the development and investigation of neural networks have become common and heated topics for the researchers in biology, mathematics, physics, and computer science. ${ }^{[27-40]}$

According to different standards of classification, neural networks can be divided into different categories. From the point of topology, neural networks can be divided into feedforward neural networks $(\mathrm{FNNs})^{[41-44]}$ and recurrent neural networks (RNNs). ${ }^{[45-65]}$ Note that the FNNs are the hierarchical structure with each layer divided by the function as the input-layer, the hidden-layer, and the output-layer. Each neuron in FNNs receives the inputs from the previous layer, and exports the information to the next layer. The information is transmitted with a fixed (or to say, single) direction without China.

${ }^{*}$ Correspondence: Shuai Li; Email: shuaili@ polyu.edu.hk; Address: Department of Computing, Hong Kong Polytechnic University, Hong Kong, 
feedback. ${ }^{[66]}$ Differing from the FNNs, the RNNs are those neural networks that possess the feedback connections of each network layers. ${ }^{[67]}$ The network structure of RNNs is more complicated than the one of FNNs. Specifically, each neuron in the RNNs exports outputs to other neurons via the connected synapses. At the same time, each neuron in the RNNs receives inputs from other neurons via the connected synapses. The input information lies on the initial states of RNNs. Then, real-time states of RNNs vary adaptively. Finally, the RNNs converge to the equilibrium states (or termed steady states), and the steady states are the outputs of the RNNs. Recently, many special classes and branches of RNNs have been developed and investigated, such as primaldual neural networks (PDNNs), ${ }^{[68-76]}$ Zhang neural networks [or termed zeroing neural networks $(\mathrm{ZNNs})^{[77-106]}$ and gradient neural networks (GNNs). ${ }^{[107-114]}$ Note that the ZNN is a special class and branch of RNNs, which is originated and extended from the research of Hopfield neural networks. The ZNNs have been proposed developed, and investigated as a systematic as well as efficient approach to solve different dynamical engineering problems in real time since 2001. ${ }^{[68,69]}$ The applications, especially the applications in robot autonomy, of those RNNs have become interesting issues for researchers and engineers. ${ }^{[15-120]}$

With the national industrialization, robot systems play an increasingly significant role in applications of modern en- gineering, life services as well as numerous fields. Robot systems have been widely used in engineering applications for cutting, welding, painting and assembly, etc. In addition, they have been widely used in life services for medical assistance, rescue, etc. Generally speaking, robot systems can be divided into serial robot manipulators (e.g., the Baxter robot $^{[121-127]}$ ), parallel robot manipulators (e.g., the Stewart platform ${ }^{[128-130]}$ ), mobile platform robots (e.g., the Mobile Kinova manipulator ${ }^{[131,132]}$ ), multirobot systems (e.g., the multiple redundant manipulators ${ }^{[133,134]}$ ), flying robots (e.g., the unmanned aerial vehicle ${ }^{[135]}$ ) and exoskeleton robots (e.g., the knee exoskeleton ${ }^{[136]}$ ). One significant issue in the research of robot systems is the motion planning and control problem. Up to now, a large number of effective approaches for robot systems have been creatively proposed and effectively employed, such as the neural networks approach, ${ }^{[137]}$ the active control approach, ${ }^{[138]}$ the robust control approach, ${ }^{[139]}$ the optimal control approach, ${ }^{[140]}$ etc. In this survey, we aim to provide a comprehensive review on the research of neural networks (or to say, neural dynamics) in robot autonomy. Specifically, the state-of-the-art research of RNNs, PDNNs, ZNNs and GNNs in different robot control problems solving are detailedly revisited and reported, with the typical applications shown in Figure 1. The readers can readily find many referenced and valuable solutions on the basis of neural networks for the robot autonomy in this paper.

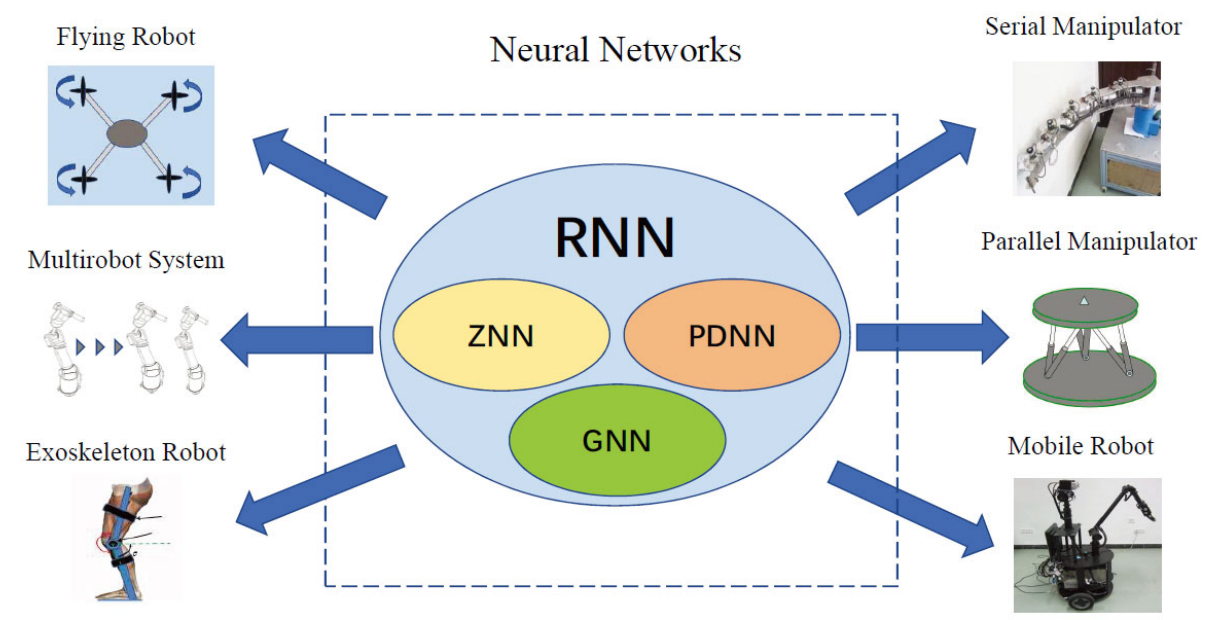

Figure 1. Applications of neural networks in robot autonomy

\section{RNN IN ROBOT AUTONOMY}

In the past three decades, by leveraging the advantages of parallelism and adaptivity as well as the free of off-line learning, the RNNs have received considerable investigation in robot autonomy. For example, Zhang and Zhang ${ }^{[116]}$ effectively solved the joint-angle as well as joint-velocity drift problems during the repeated motion of robot manipulators, by propos- ing a drift-free method in acceleration-level limited by the linear equality constraint. Noth that the effectiveness was detailedly analysed on the basis of the theory of a secondorder system. Such a method proposed in ${ }^{[116]}$ is afterwards formulated as the quadratic programming (QP), and solved by corresponding RNNs. In, ${ }^{[141]}$ a novel dual-manipulator repetitive motion generation strategy was proposed by the 
neural dynamics approach to handle and address a joint-drift problem of the humanoid robot. Specifically, based on such a neural dynamics approach, a performance index for repetitive motion was effectively utilized in,${ }^{[141]}$ and then such a performance index was integrated as the $\mathrm{QP}$ formulation with the time-varying constraints. Specifically it was named as the time-varying constrained scheme. Such a scheme can effectively produce a repeated motion of two manipulators of the humanoid robot. Besides, it also can control two manipulators to move to a desired position, which was finally solved by a RNN to achieve the optimal solutions. In addition, Zhang et al. ${ }^{[142]}$ employed a virtual plane method to produce a analytical solution for the motion of the robot head. Then, a QP-based method was exploited to formulate the coordinated dual-arm motion. By utilizing a simplified RNN, the optimal solution was finally found. Mohammed and $\mathrm{Li}^{[128]}$ investigated and formulated the kinematics control for the Stewart platform as the QP with constraint. Note that Karush-Kuhn-Tucker conditions of problem were attained via considering the primal one in dual space, and afterwards an RNN was developed to handle such a optimization problem. Detailed theoretical analyses in $^{[128]}$ prove the global convergence of such a neural network to the optimal solution according to the defined performance index. In 2017, Li et al. ${ }^{[143]}$ identified two major limitations of the RNN solutions for the robot manipulator autonomy, that is the accumulation of position error as well as the convex restriction of projection set. The proposed method in reference ${ }^{[143]}$ overcame the above mentioned limitations by developing modified neural network models, which enables nonconvex sets of projection operations, and the position error will not accumulate over time under the influence of the noise. Differing from many works the corresponding RNNs are utilized to address time sequences, the corresponding method has the advantages of model-based as well as training-free. It makes it feasible to obtain rapid tracking of reference signals. In, ${ }^{[144]}$ three different RNNs and three different numerical methods was investigated, developed, and compared to solve a repetitive motion planning (RMP) scheme for remedying joint-drift problems of redundant robot manipulators. Three RNNs presented in $^{[144]}$ are recurrent and real time, and they do not need to be trained in advance. $\mathrm{Li}$ et al. ${ }^{[145]}$ proposed a novel RNN to handle the redundancy of robots for efficient kinematics problem under the influence of polynomial-type noises. By taking advantage of high-order derivative properties of polynomialtype noises, the devised neural network model was developed to remove the negative influence of noises, and regain the effective tracking of reference trajectories in high accuracy. To solve the joint drift phenomenon that might lead to the failure of the given task, or even worse, the damage of the robot, a finite time varying parameter RNN was introduced and developed in. ${ }^{[146]}$ A QP-type joint drift-free solution was proposed, which consists of an optimization performance index and a velocity-level kinematics equation. Note that a feedback information was added to the kinematics equation as a constrained equality. In addition, a novel joint drift-free solution considering feedback was achieved. Then, a novel RNN model was developed to solve the proposed scheme, and the related finite-time convergence theorem was given. Specifically, the superiorities of the proposed RNN in ${ }^{[146]}$ the real-time computation, the exponential convergence, as well as the capacity to remove initial errors. Moreover, Jin et al. ${ }^{[147]}$ revisited the existing RNN and it related models for addressing zero-finding, such as the inversion of matrix, with time-varying parameters from control perspective, and then formulated as a control-theoretical framework. Afterwards, the constraints of activated functions of RNN and the related models were presented, and handled by taking advantage of control techniques. Besides, the gradient-based RNN, as a typical solution for zero-finding, were represented to handle the dynamic issues in manners free of errors as well as matrix inversions. The research in ${ }^{[147]}$ provided a systematic and general method on using control techniques to develop the corresponding RNN and the related models for robustly as well as accurately handling algebraic equations and the robot autonomy problems. More detailed research by utilizing the RNNs with application to robot autonomy can also be refereed in the state-of-the-art works. ${ }^{[148-150]}$

\section{PDNN IN ROBOT AUTONOMY}

Many special classes and branches of RNNs have been introduced and investigated for the robot kinematic as well as dynamic autonomy, e.g., the Lagrangian neural networks and the PDNNs. ${ }^{[151]}$ In particular, Zhang et al. ${ }^{[68]}$ proposed a dual neural network (i.e., a special case of PDNNs) for the bi-criteria kinematics issue of robot manipulators. In order to eliminate discontinuity of minimum infinity-norm solutions, the kinematics control issue was reformulated as the bi-criteria of infinity and Euclidean norms in. ${ }^{[68]}$ Note that physical constraints, like joint-angle limit as well as jointvelocity limits were incorporated in the kinematics control solution. Such a dual neural network was proven to converge to the optimal solutions globally in a bi-criteria sense, and was illustrated to be effective for the the PA10 robot autonomy in. ${ }^{[68]}$ In addition, a dual neural network was developed in $^{[69]}$ for the real-time joint-torque optimization of redundant robot manipulators corresponding to the global energy minimization of robot manipulators. Differing from existing computational schemes on the inverse kinematics, a dual neural network was introduced at acceleration level to handle 
redundancy issue of limited joint-range robots. Note that such a dual neural network possesses a quite simple structure with one layer of neurons. It was proved to converge to the optimal solutions globally as well as exponentially in. ${ }^{[69]}$ Such a dual neural network was finally simulated with a PUMA 560 robot manipulator to substantiate effectiveness. In 2004, Zhang and Wang ${ }^{[71]}$ proposed and developed a new dual neural network with application to kinematics autonomy of redundant robot manipulators with the ability of obstacle avoidance. Note that the requirement of obstacle avoidance was represented by the dynamical inequality limits, an improved problem formulation was presented in. ${ }^{[71]}$ On the basis of the above improved problem formulation, a novel dual neural network was introduced for solving real-time collision-free problem of inverse kinematics. Such a proposed new dual neural network was applied to the autonomy of a PA10 robot manipulator with a point obstacle as well as a window-shaped obstacle. In 2007, the neural computation of real-time solution of the matrix-inverse problems was investigated in. ${ }^{[152]}$ The basic concepts of primal neural network and power-sigmoid activation function were thus formally proposed for the general neural computation of matrix inverse. Different activation functions were examined and presented $\mathrm{in}^{[152]}$ for the superior convergence as well as robustness of the system involved. In addition to the singular case, the reference ${ }^{[152]}$ investigated a robotic example, that is, inverse kinematic autonomy of redundant robots by using real-time pseudo-inverse computation. Chen and Zhang ${ }^{[153]}$ proposed a novel minimum jerk norm solution with the obstacle-avoidance constraint with the application to a redundant manipulator, of which its joint jerks keep bounded for the human-friendly robot autonomy. For the aim of superior tracking performance of the redundant manipulator, the presented jerk bounded MJN solution in ${ }^{[153]}$ was further improved by feedback information. Moreover, the effectiveness of the obstacle avoidance of the solution was proven by a variable-magnitude escape-jerk theorem. Then, the proposed solution was formulated into the dynamical QP which is then handled by a special kind of PDNNs. In 2017, a hybrid multi-objective (HMO) solution was novelly developed in ${ }^{[76]}$ to simultaneously achieve four objectives, i.e., the specified main task for end-effector, obstacle avoidance, joint-physical limits avoidance, as well as repetitive motion of robot manipulators. Afterwards, such an HMO solution in $^{[76]}$ was formulated into a dynamical QP with the optimal solution of the dynamical QP problem found by a special kind of PDNNs and also by a numerical algorithm implemented on the computer. In, ${ }^{[154]}$ a jerk-level synchronous repetitive motion solution was introduced to handle the jointangle-drift issue, and obtain the synchronous autonomy of a dual manipulators of redundant robot. Such a solution in ${ }^{[154]}$ was solved at joint-jerk level making all the joint variables, i.e., joint angles, joint velocities as well as joint accelerations, smooth and bounded. In addition, different types of dynamics algorithms, that is the gradient-type as well as zeroing-type dynamics algorithms, to design the repetitive motion variable vectors, were detailedly shown with circuit schematics. Afterwards, the presented solution in ${ }^{[154]}$ was formulated into two dynamical QPs, and then integrated into unified dynamical QP for the synchronous autonomy of a dual manipulator of robot system. Note that the optimal solution for UDQP was successfully achieved via a kind of PDNNs. More detailed research by utilizing the PDNNs with application to robot autonomy can also be refereed in the state-of-the-art works. ${ }^{[155-157]}$

\section{ZNN IN ROBOT AUTONOMY}

Remarkably, as a new class of RNNs, Zhang neural networks (or to say, zeroing neural networks, ZNN) can handle problems with multiple state dimensions. ${ }^{[158]}$ Specifically, such class of RNNs can zero out each element of error function in the neural dynamics manner, ${ }^{[159]}$ which is thus deemed as a systematic as well as effective methodology to solve different real-time robotic issues. ${ }^{[160-162]}$ For example, to effectively handle inverse kinematics issue of redundant robots, the redundancy-resolution solutions were investigated in. ${ }^{[84]}$ The first one was solved at the joint-velocity level, and the second one was solved at the joint-acceleration level. Both the solutions in ${ }^{[84]}$ were formulated into a dynamical QP. Then, the ZNN was introduced and presented for real-time solution of the related QP. For the purpose of accurate solution of the real-time inverse kinematics issue for the mobile robots, an interesting $\mathrm{ZNN}$ is introduced and developed by Xiao and Zhang. ${ }^{[85]}$ It was theoretically proven that the corresponding model of $\mathrm{ZNN}$ in ${ }^{[85]}$ can globally as well as exponentially converge to the solution of the real-time inverse kinematics problem for mobile robots. Moreover, kinematics equations of a mobile platform as well as a manipulator were integrated as one robot system, and thus the corresponding solution can coordinate simultaneously wheels as well as manipulator to successfully achieve a desired end-effector job. In, ${ }^{[163]}$ the authors made progress along the direction by introducing a modified zeroing neural network (i.e., the MZNN) model for solving the time-varying QP problem. Noth that an original ZNN model and a GNN model are used to compare with the presented MZNN model. Note that detailed theoretical analyses in ${ }^{[163]}$ prove that the presented MZNN model globally and exponentially converges to the exact real-time solution of the real-time QP without measurement noise In addition, under the influence of the 
measurement noises, such a presented MZNN model would possess a satisfactory performance. Moreover, Jin et al. ${ }^{[164]}$ presented and investigated an integration-enhanced $\mathrm{ZNN}$ and the related model for solving time-varying matrix inversion Note that theoretical analyses in ${ }^{[164]}$ prove that the presented integration-enhanced ZNN model possesses the global and exponential convergence property. In addition, under the influence of different kinds of noises, such an integrationenhanced ZNN together with the related model was proven to possess an improved performance. Note that no matter how large the matrix-form constant noise is, such an integrationenhanced ZNN model in ${ }^{[164]}$ can converge to the theoretical solution. In addition, the residual errors of the presented integration-enhanced ZNN model would be arbitrarily small as for the time-varying noises as well as the random noises. Finally, such an integration-enhanced ZNN model was used to the autonomy problem handling of robots. For the research of discrete-time ZNN, a novel Taylor numerical differentiation formula was introduced for the discretization of the continuous-time $\mathrm{ZNN}$ in ${ }^{[165]}$ to achieve higher computational accuracy. On the basis of such a Taylor numerical differentiation formula, the corresponding Taylor-type discrete-time ZNN models were then introduced and discussed to perform the real-time dynamic equality-constrained QP. For comparison purpose, the Euler-type discrete-time ZNN models as well as the Newton iteration together with their links being found, were also shown in. ${ }^{[165]}$ It was shown in ${ }^{[165]}$ that the steady-state residual errors of the presented Taylor-type ZNN models, Euler-type ZNN models, as well as Newton iteration have the patterns of $O\left(h^{3}\right), O\left(h^{2}\right)$, as well as $O(h)$, respectively, with $h$ being the sampling gap. Finally, such a Taylortype discrete-time $\mathrm{ZNN}$ model was applied to the control problem solving of robot manipulators. Moreover, Chen and Zhang ${ }^{[166]}$ proposed a novel robust ZNN model for handling the inverse kinematics issue of the mobile robots. Differing from most $\mathrm{ZNN}$ works on the basis of the assumption that neural networks are free of external disturbances, four common forms of time-varying disturbances suppressed by the presented robust ZNN model were developed in. ${ }^{[166]}$ Note that theoretical analyses of anti-disturbance performance therein were presented to prove the effectiveness as well as robustness of the presented robust ZNN model with timevarying disturbances suppressed for handling the inverse kinematics issue of mobile robots. In addition, to overcome two major limitations in traditional Jacobian-matrix-pseudoinverse method, Chen et al. ${ }^{[167]}$ proposed an interesting Jacobian- matrix-adaption (JMA) approach for the tracking autonomy of robots via the ZNN design process. Differing from most $\mathrm{ZNN}$ works requiring the information of the known robot model, the proposed JMA approach used only the input-output information to control the robot with unknown model. The solution on the basis of such a JMA approach in ${ }^{[167]}$ successfully transforms the internal, implicit and unmeasurable model information to the external, explicit as well as measurable input-output information. In, ${ }^{[168]}$ the authors proposed a novel control method for the controlling knee exoskeleton robot with the real-time inertial and viscous coefficients. Note that the controller was designed on the basis of the ZNN approach and utilized twice Zhang function so as to make the tracking error of joint angle exponentially converge to zero. In, ${ }^{[135]}$ the authors proposed and investigated a varying-parameter convergent neural dynamic (VP-CND) autonomy approach by exploiting ZNN design approach to stably control the position and attitude angles of an unmanned aerial vehicle (specifically, a flying robot). The proposed VP-CND autonomy approach for the flying robot in $^{[135]}$ not only can track time-varying desired values but also possesses super-exponential convergence performance. Jin et al. ${ }^{[133]}$ proposed and developed a distributed solution for the cooperative motion generation in a distributed network of multiple redundant robots (specifically, multirobot system). In order to suppress the noises originating from communication interferences and computational errors, a noise-tolerant $\mathrm{ZNN}$ is constructed to solve the QP in real-time. Note that the theoretical as well as the simulative results shown that, in the presence of noise, the proposed distributed scheme in $^{[133,134]}$ with the aid of noise-tolerant ZNN model has a satisfactory performance.

\section{GNN IN ROBOT AUTONOMY}

Another special class of RNN, i.e., the GNN, has been introduced and developed as an effective option for the real-time scientific problems handling including the robotics redundancy resolution problem. For example, in 2005, a gradient based neural dynamics system for matrix inversion was revisited by examining different activation functions as well as various implementation errors in. ${ }^{[107]}$ Then, a general GNN for matrix inversion was thus introduced which was constructed by utilizing the monotonically-increasing odd activation functions. For the aim of superior convergence and robustness of the presented system, the power-sigmoid activation function was preferred to be in use. In addition to investigating the singular case, the reference ${ }^{[107]}$ also presented an example on inverse kinematic autonomy of redundant robots via real-time pseudo-inverse solution. In 2008, a QP-type method was employed for repetitive motion generation of robot manipulators together with the joint-physical limits considered by Chen et al. ${ }^{[169]}$ In order to illustrate the effectiveness of such a QP-type repetitive motion generation solution, different kinds of multi-link planar manip- 
ulators are used to perform square end-effector trajectories through different simulations. Note that theoretical analysis based on GNN therein was conducted to prove the efficacy of the presented scheme. In 2011, the performance of a GNN, which was developed for handling static problems intrinsically, was introduced as well as analyzed in the situation of time-varying coefficients in. ${ }^{[111]}$ Specifically, it was theoretically shown that the GNN for real-time solution of time-varying quadratic minimization as well as the corresponding QP problems could only approximately approach the time-varying theoretical solution, instead of converging exactly. In other words, the steady-state error between the GNN solution and the theoretical solution can not decrease to 0 . For better understanding the situation, the upper bound of such an error was estimated firstly, and then the global and exponential convergence rate was investigated for such a GNN when approaching the error bound. In 2016, to avoid the Jacobian inversion in the conventional pseudo-inverse solution effectively, and also to achieve the solution of the minimum two-norm position error to the inverse kinematics of the mobile robot, a novel inverse-free solution using the GNN design approach was introduce and developed in. ${ }^{[170]}$ Note that the inversion of Jacobian matrix is usually required in the pseudo-inverse approach as handling the robotic autonomy problem, which is computationally intensive, especially for the complex mobile robots. By using the advantages of the mutual coordination effect between a mobile platform with two omnidirectional driving wheels and a six-joint manipulator, the integrated kinematics of a robot system was derived and developed therein to coordinate the motions of the platform as well as the manipulator. Moreover, the presented inverse-free solution in ${ }^{[170]}$ with different values of design parameter as well as the conventional pseudo-inverse solution were conducted for comparison on the basis of the robot system for specific tracking jobs. In addition, Chen and Zhang ${ }^{[154]}$ proposed a novel jerk-level synchronous repetitive motion solution to address the joint drift problem, and obtain the synchronous autonomy of a dual manipulators of robot system. Note that the corresponding solution was resolved at joint-jerk level making the joint variables, i.e. the joint angles, the joint velocities as well as the joint accelerations, smooth and bounded. In, ${ }^{[154]}$ different kinds of dynamics algorithms, i.e. gradient-type (G-type) as well as zeroingtype (Z-type) dynamics algorithms, on the basis of the GNN and the ZNN design approaches respectively, for designing repetitive motion vectors, were shown together with circuit schematics.

\section{Conclusion}

In this review, a comprehensive survey of the research on neural networks in robot autonomy has been presented. Specifically, the state-of-the-art research of RNNs, PDNNs, ZNNs and GNNs in different robot control problems have been detailedly revisited and reported. In addition, the readers and researchers can readily find many referenced solutions on the basis of neural networks for the robot control problem in this review. For future directions of the research on neural networks in robot autonomy, we now provide some prospective outlooks.

- More classes and branches of advanced neural networks would be developed and investigated for solving control problems of robots in different applications.

- The research of complexity, stability and robustness of neural networks would be detailed investigated during the applications to advanced robots in complex environment.

- The corresponding circuit systems of related neural networks would be developed, implemented and applied to the robot autonomy hardware systems in industry.

\section{ACKNOWLEDGEMENTS}

This work is supported by the National Natural Science Foundation of China (with numbers 61401385 and 61702146 ), by Hong Kong Research Grants Council Early Career Scheme (with number 25214015), by Departmental General Research Fund of Hong Kong Polytechnic University (with number G.61.37.UA7L), and also by PolyU Central Research Grant (with number G-YBMU).

\section{REFERENCES}

[1] Narendra KS, Parthasarathy K. Identification and control of dynamical systems using neural networks. IEEE Transactions on Neural Networks. 1990; 1(1): 4-26. PMid:18282820. https://doi.org/ 10.1109/72.80202

[2] Cichocki A, Unbehauen R. Neural networks for solving systems of linear equation and related problems. IEEE Transactions on Circuits and Systems. 1992; 39(2): 124-138. https://doi.org/10.1109/ 81.167018

[3] Wang J, Wu G. A multilayer recurrent neural network for solving continuous-time algebraic Riccati equations. Neural Networks. 1998; 11: 939-950. https://doi .org/10.1016/S0893-6080(98) 000 34-3

[4] Chu YC, Huang J. A neural-network method for the nonlinear ser- 
vomechanism problem. IEEE Transactions on Neural Networks. 1999; 10(6): 1412-1423. PMid:18252642. https://doi.org/10 $.1109 / 72.809086$

[5] Zhang Y, Wang J. Recurrent neural networks for nonlinear output regulation. Automatica. 2001;37(8): 1161-1173. https://doi.or g/10.1016/S0005-1098(01)00092-9

[6] Xia Y, Wang J, Hung DL. Recurrent neural networks for solving linear inequalities and equations. IEEE Transactions on Circuits and Systems I: Fundamental Theory and Applications. 1999; 46(4): 452462. https://doi.org/10.1109/81.754846

[7] Xia Y, Wang J. A recurrent neural network for solving linear projection equations. Neural Networks. 2000; 13: 337-350. https: //doi.org/10.1016/S0893-6080(00)00019-8

[8] Zhang Y, Jiang D, Wang J. A recurrent neural network for solving Sylvester equation with time-varying coefficients. IEEE Transactions on Neural Networks. 2002; 13(5): 1053-1063. PMid:18244503. https://doi.org/10.1109/TNN. 2002.1031938

[9] Jiang X, Li S. BAS: beetle antennae search algorithm for optimization problems. International Journal of Robotics and Control. 2018; 1(1): 1-5.

[10] Nakazawa T, Kulkarni DV. Wafer map defect pattern classification and image retrieval using convolutional neural network. IEEE Transactions on Semiconductor Manufacturing. 2018; 31(2): 309-314. https://doi.org/10.1109/TSM.2018.2795466

[11] Anthimopoulos M, Christodoulidis S, Ebner L, Christe A, Mougiakakou S. Lung pattern classification for interstitial lung diseases using a deep convolutional neural network. IEEE Transactions On Medical Imaging. 2016; 35(5): 1207-1216. PMid:26955021. https://doi.org/10.1109/TMI. 2016.2535865

[12] Han Z, Liu Z, Vong CM, Liu YS, Bu S, Han J, et al. Deep spatiality: unsupervised learning of spatially-enhanced global and local 3D features by deep neural network with coupled softmax. IEEE Transactions on Image Processing. 2018; 27(6): 3049-3063. PMid:29993805. https://doi.org/10.1109/TIP.2018.2816821

[13] Dey D, Chatterjee B, Dalai S, Munshi S, Chakravorti S. A deep learning framework using convolution neural network for classification of impulse fault patterns in transformers with increased accuracy. IEEE Transactions on Dielectrics and Electrical Insulation. 2017; 24(6) 3894-3897. https://doi.org/10.1109/TDEI. 2017.006793

[14] Zhang Y, Chen D, Jin L, Wang Y, Luo F. Twice-pruning aided WASD neuronet of bernoulli-polynomial type with extension to robust classification. In Proceedings of International Conference on Dependable, Autonomic and Secure Computing. 2013; 334-339. https://doi.org/10.1109/DASC.2013.85

[15] Chen D, Qiao T, Tan H, Li M, Zhang Y. Solving the problem of runge phenomenon by pseudoinverse cubic spline. In Proceedings of International Conference on Computational Science and Engineering. 2014; 1226-1231. https://doi.org/10.1109/CSE. 2014. 237

[16] Hu X, Feng G, Duan S, Liu L. A memristive multilayer cellular neural network with applications to image processing. Ieee Transactions on Neural Networks and Learning Systems. 2017; 28(8): 1889-1901. PMid:27187973. https ://doi .org/10.1109/TNNLS.2016.255 2640

[17] Kung J, Kim D, Mukhopadhyay S. On the impact of energy-accuracy tradeoff in a digital cellular neural network for image processing. IEEE Transactions on Computer-Aided Design of Integrated Circuits and Systems. 2015; 34(7): 1070-1081. https ://doi .org/10.110 9/TCAD. 2015.2406853

[18] Vahed A, Omlin CW. A machine learning method for extracting symbolic knowledge from recurrent neural networks. Neural Computation. 2004; 16(1): 59-71. PMid:15006023. https://doi.org/ $10.1162 / 08997660460733994$
[19] Xia Y, Wang J. A one-layer recurrent neural network for support vector machine learning. IEEE Transactions on Systems, Man, and Cybernetics, Part B: Cybernetics. 2004; 34(2): 1261-1269. https://doi.org/10.1109/TSMCB.2003.822955

[20] Li S, Li Y, Wang Z. A class of finite-time dual neural networks for solving quadratic programming problems and its $k$-winners-take-all application. Neural Networks. 2013; 39: 27-39. PMid:23334164. https://doi.org/10.1016/j.neunet.2012.12.009

[21] Li S, Kong R, Guo Y. Cooperative distributed source seeking by multiple robots: algorithms and experiments. IEEE/ASME Transactions on mechatronics. 2014; 19(6): 1810-1820. https ://doi.org/10 .1109/TMECH. 2013. 2295036

[22] Li S, Chen S, Liu B, Li Y, Liang Y. Decentralized kinematic control of a class of collaborative redundant manipulators via recurrent neural networks. Neurocomputing. 2012; 91: 1-10. https: //doi.org/10.1016/j.neucom.2012.01.034

[23] Zhang Y, Chen D, Yin Y, Guo D, Xie Q. ZG tracking control of Lu system with multiple inputs and with division-by-zero problem solved. In Proceedings of Chinese Control Conference. 2014; 3477 3482. https://doi.org/10.1109/ChiCC. 2014.6895516

[24] Li J, Mao M, Zhang Y, Chen D, Yin Y. ZD, ZG and IOL controllers and comparisons for nonlinear system output tracking with DBZ problem conquered in different relative-degree cases. Asian Journal of Control. 2017; 19(4): 1482-1495. https ://doi.org/10.1002/ asjc. 1460

[25] Li S, Cui H, Li Y, Liu B, Lou Y. Decentralized control of collaborative redundant manipulators with partial command coverage via locally connected recurrent neural networks. Neural Computing and Applications. 2012; 23(3-4): 1051-1060. https ://doi.org/10.1 007/s00521-012-1030-2

[26] Shi Y, Qiu B, Chen D, Li J, Zhang Y. Proposing and validation of a new four-point finite-difference formula with manipulator application. IEEE Transactions on Industrial Informatics. 2018; 14(4): 1323-1333. https://doi .org/10.1109/TII . 2017.2787799

[27] Li S, Liu B, Li Y. Selective positive-negative feedback produces the winner-take-all competition in recurrent neural networks. IEEE transactions on neural networks and learning systems. 2013; 24(2): 301309. PMid:24808283. https://doi.org/10.1109/TNNLS. 2012 .2230451

[28] Zhang Z, Fu T, Yan Z, Jin L, Xiao L, Sun Y, et al. A varying-parameter convergent-differential neural network for solving joint-angular-drift problems of redundant robot manipulators. IEEE/ASME Transactions on Mechatronics. 2018. (In press). https://doi .org/10.1 109/TMECH. 2018. 2799724

[29] Zhang Z, Lu Y, Zheng L, Li S, Yu Z, Li Y. A new varying-parameter convergent-differential neural-network for solving time-varying convex $\mathrm{QP}$ problem constrained by linear-equality. IEEE Transactions on Automatic Control. 2018. (In press). https ://doi .org/10.1 109/TAC. 2018. 2810039

[30] Zhang Z, Zheng L, Jian W, Mao Y, Lu W, Xiao L. A new varyingparameter recurrent neural-network for online solution of timevarying sylvester equation. IEEE Transactions on Cybernetics. 2017 (In press). https://doi.org/10.1109/TCYB. 2017.2760883

[31] Zhang Z, Lin Y, Li S, Li Y, Luo Y. Tri-criteria optimizationcoordination-motion of dual redundant robot manipulators for complex path planning. IEEE Transactions on Control Systems Technology. 2017. (In press). https ://doi .org/10.1109/TCST . 201 7.2709276

[32] Cheng CH, Li S, Kadry S. Mind-wave controlled robot: an arduino robot simulating the wheelchair for paralyzed patients. International Journal of Robotics and Control. 2018. (In press). https : //doi.org/10.5430/ijrc.v1n1p6 
[33] Jiang X, Li S. Plume front tracking in unknown environments by estimation and control. IEEE Transactions on Industrial Informatics. 2018. (In press). https://doi .org/10.1109/TII. 2018. 28312 25

[34] Zhang Y, Li W, Guo D, Ke Z. Different Zhang functions leading to different ZNN models illustrated via time-varying matrix square roots finding. Expert Systems with Applications. 2013; 40(11): 4393-4403. https://doi.org/10.1016/j.eswa.2013.01.045

[35] Li S, Guo Y. Distributed source seeking by cooperative robots: All-to-all and limited communications. In Proceedings of International Conference on Robotics and Automation. 2012; 1107-1112. https://doi.org/10.1109/ICRA. 2012.6224713

[36] Xiao L, Zhang Y. Two new types of Zhang neural networks solving systems of time-varying nonlinear inequalities. IEEE Transactions on Circuits and Systems I: Regular Papers. 2012; 59(10): 2363-2373. https://doi.org/10.1109/TCSI . 2012.2188944

[37] Li S, Guo Y. Distributed consensus filter on directed switching graphs. International Journal of Robust and Nonlinear Control. 2015; 25(13): 2019-2040. https://doi.org/10.1002/rnc. 3187

[38] Li S, Guo Y. Dynamic consensus estimation of weighted average on directed graphs. International Journal of Systems Science. 2015; 46(10): 1839-1853. https://doi.org/10.1080/00207721.201 3.837541

[39] Ma W, Cheng H, Li Z, et al. Time-varying stiffness tracking control of knee exoskeleton. In Proceedings of International Conference on Robotics and Biomimetics. 2017; 1665-1669.

[40] Li Z, Yin Z. Tracking control of knee exoskeleton with time-varying model coefficients under compliant interaction. In Proceedings of International Conference on Chinese Control Conference. 2017; 66026607. https://doi.org/10.23919/ChiCC. 2017.8028403

[41] Guo D, Zhang Y, Xiao Z, et al. Common nature of learning between BP-type and Hopfield-type neural networks. Neurocomputing. 2015; 167: 578-586. https://doi.org/10.1016/j.neucom. 2015.04 .032

[42] Zhang Y, Yin Y, Guo D, Yu X, Xiao L. Cross-validation based weights and structure determination of Chebyshev-polynomial neural networks for pattern classification. Pattern Recognition. 2014; 47: 34143428. https://doi.org/10.1016/j. patcog. 2014.04.026

[43] Zhang Y, Guo D, Li Z. Common nature of learning between backpropagation and Hopfield-type neural networks for generalized matrix inversion with simplified models. IEEE Transactions on Neural Networks and Learning Systems. 2013; 24(4): 579-592. PMid:24808379. https://doi.org/10.1109/TNNLS. 2013.223 8555

[44] Zhang Y, Wang Y, Li W, Chou Y, Zhang Z. WASD algorithm with pruning-while-growing and twice-pruning techniques for multi-input Euler polynomial neural network. International Journal on Artificial Intelligence Tools. 2016; 25(02): 1650007. https ://doi .org/10 $.1142 / \mathrm{S} 021821301650007 \mathrm{X}$

[45] Wang J. Recurrent neural networks for solving linear matrix equations. Computers and Mathematics with Applications. 1993; 26(9): 23-34. https://doi.org/10.1016/0898-1221 (93)90003-E

[46] Wang J. Recurrent neural networks for computing pseudoinverses of rank-deficient matrices. SIAM Journal on Scientixc Computing. 1997; 18(5): 1479-1493.

[47] Li S, He J, Rafique MU, Li Y. Distributed recurrent neural networks for cooperative control of manipulators: A game-theoretic perspective. IEEE Transactions on Neural Networks and Learning Systems. 2016; 14(3): 658-667.

[48] Li S, Zhang Y, Jin L. Kinematic control of redundant manipulators using neural networks. IEEE Transactions on Neural Networks and Learning Systems. 2017; 28(10): 2243-2254. PMid:27352398. https://doi.org/10.1109/TNNLS. 2016.2574363

[49] Tang WS, Wang J. A recurrent neural network for minimum infinitynorm kinematic control of redundant manipulators with an improved problem formulation and reduced architecture complexity. IEEE Transactions on Systems, Man, and Cybernetics, Part B: Cybernetics. 2001; 31(1): 98-105. PMid:18244770. https://doi .org/10.110 $9 / 3477.907567$

[50] Zhang YN, Ge SZ. Design and analysis of a general recurrent neural network model for time-varying matrix inversion. IEEE Transactions on Neural Networks. 2005; 16(6): 1477-1490.

[51] Jin L, Li S, Hu B, Liu M, Yu J. Noise-suppressing neural algorithm for solving time-varying system of linear equations: a control-based approach. IEEE Transactions on Industrial Informatics. 2018. (In press). https://doi.org/10.1109/TII.2018.2798642

[52] Jin L, Li S, Hu B, Yi C. Dynamic neural networks aided distributed cooperative control of manipulators capable of different performance indices. Neurocomputing. 2018; 291: 50-58.

[53] Jin L, Li S, Yu J, He J. Robot manipulator control using neural networks: a survey. Neurocomputing. 2018; 285: 23-34.

[54] Zhang Y, Li S, Jiang X. Near-optimal control without solving HJB equations and its applications. IEEE Transactions on Industrial Electronics. 2018; 65(9): 7173-7184. https://doi .org/10.1109/TI E. 2018.2793233

[55] Jin L, Li S, Luo X, Li Y, Qin B. Neural dynamics for cooperative control of redundant robot manipulators. IEEE Transactions on Industrial Informatics. 2018. (In press). https://doi .org/10.110 9/TII. 2018. 2789438

[56] Li J, Zhang Y, Li S, Mao M. Neural dynamics for cooperative control of redundant robot manipulators. IEEE Transactions on Industrial Informatics. 2018. (In press). https://doi.org/10.1109/TII . 2017.2780892

[57] Li S, Zhou M, Luo X. Modified primal-dual neural networks for motion control of redundant manipulators with dynamic rejection of harmonic noises. IEEE Transactions on Neural Networks and Learning Systems. 2017. (In press). https://doi.org/10.1109/TNNL S. 2017.2770172

[58] Xiao L, Zhang Z, Zhang Z, Li W, Li S. Design, verification and robotic application of a novel recurrent neural network for computing dynamic Sylvester equation. Neural Networks. 2018; 105: 185-196. PMid:29870926. https://doi.org/10.1016/j.neunet. 2018. 05.008

[59] Xiao L, Zhang Z, Li S. Solving time-varying system of nonlinear equations by finite-time recurrent neural networks with application to motion tracking of robot manipulators. IEEE Transactions on Systems, Man, and Cybernetics: Systems. 2018. (In press). https://doi .org/10.1109/TSMC . 2018.2836968

[60] Xiao L, Liao B, Li S, Zhang Z, Ding L, Jin L. Design and analysis of FTZNN applied to the real-time solution of a nonstationary Lyapunov equation and tracking control of a wheeled mobile manipulator. IEEE Transactions on Industrial Informatics. 2018; 14(1): 98-105. https://doi.org/10.1109/TII.2017.2717020

[61] Xiao L. A finite-time recurrent neural network for solving online time-varying Sylvester matrix equation based on a new evolution formula. Nonlinear Dynamics. 2017; 90(3): 1581-1591. https: //doi.org/10.1007/s11071-017-3750-4

[62] Xiao L. Accelerating a recurrent neural network to finite-time convergence using a new design formula and its application to time-varying matrix square root. Journal of the Franklin Institute. 2017; 354(13): 5667-5677. https://doi.org/10.1016/j.jfranklin.2017.0 6.012

Published by Sciedu Press 
[63] Xiao L, Zhang Y, Liao B, Zhang Z, Ding L, Jin L. A velocity-level bi-criteria optimization scheme for coordinated path tracking of dual robot manipulators using recurrent neural network. Frontiers in Neurorobotics. 2017; 11(45): 1-7. https://doi.org/10.3389/fnbo t. 2017.00047

[64] Ding L, Xiao L, Liao B, Lu R, Peng H. An improved recurrent neural network for complex-valued systems of linear equation and its application to robotic motion tracking. Frontiers in Neurorobotics. 2017; 11(45): 1-8.

[65] Zhang Y, Tan Z, Chen K, Yang Z, Lv X. Repetitive motion of redundant robots planned by three kinds of recurrent neural networks and illustrated with a four-link planar manipulator's straight-line example. Robotics and Autonomous Systems. 2009; 57(6-7): 645-651.

[66] Zhang Y, Qu L, Liu J, Guo D, Li M. Sine neural network (SNN) with double-stage weights and structure determination (DS-WASD). Soft Computing. 2016; 20(1): 211-221. https://doi.org/10.1007/ s00500-014-1491-6

[67] Wang J. Recurrent neural networks for computing pseudoinverses of rank-deficient matrices. SIAM Journal on Scientific Computing. 1997; 18(5): 1479-1493. https://doi.org/10.1137/S1064827 594267161

[68] Zhang Y, Wang J, Xu Y. A dual neural network for bi-criteria kinematic control of redundant manipulators. IEEE Transactions on Robotics and Automation. 2002; 18(6): 923-931. https://doi org/10.1109/TRA.2002.805651

[69] Zhang Y, Wang J. A dual neural network for constrained joint torque optimization of kinematically redundant manipulators. IEEE Transactions on Systems, Man, and Cybernetics, Part B (Cybernetics). 2002; 32(5): 654-662. PMid:18244869. https://doi .org/10.1109/TS MCB. 2002.1033184

[70] Zhang Y, Wang J, Xia Y. A dual neural network for redundancy resolution of kinematically redundant manipulators subject to joint limits and joint velocity limits. IEEE Transactions on Neural Networks. 2003; 14(3): 658-667. PMid:18238046. https://doi.org/10.1 109/TNN. 2003.810607

[71] Zhang Y, Wang J. Obstacle avoidance for kinematically redundant manipulators using a dual neural network. IEEE Transactions on Systems, Man, and Cybernetics, Part B: Cybernetics. 2004; 34(1): 752-759. https://doi .org/10.1109/TSMCB . 2003.811519

[72] Zhang Y, Ge SS, Lee TH. A unified quadratic-programming-based dynamical system approach to joint torque optimization of physically constrained redundant manipulators. IEEE Transactions on Systems, Man, and Cybernetics, Part B: Cybernetics. 2004; 34(5): 2126-2132. https://doi.org/10.1109/TSMCB. 2004.830347

[73] Zhang Y. A set of nonlinear equations and inequalities arising in robotics and its online solution via a primal neural network. Neurocomputing. 2006; 70(1-3): 513-524.

[74] Zhang Y, Cai B, Zhang L, Li K. Bi-criteria velocity minimization of robot manipulators using a linear variational inequalities-based primal-dual neural network and PUMA560 example. Advanced Robotics. 2008; 22(13-14): 1479-1496.

[75] Zhang Z, Chen S, Li S. Compatible convex-nonconvex constrained QP-based dual neural networks for motion planning of redundant robot manipulator. IEEE Transactions on Control Systems Technology. 2017. (In press). https://doi.org/10.1109/TCST . 2018. 2799990

[76] Chen D, Zhang Y. A hybrid multi-objective scheme applied to redundant robot manipulators. IEEE Transactions on Automation Science and Engineering. 2017; 14(3): 1337-1350. https://doi.org/10 .1109/TASE. 2015.2474157

[77] Zhang Y, Fan Z, Li Z. Zhang neural network for online solution of time-varying Sylvester equation. In Proceedings of International
Symposium on Intelligence Computation and Applications. 2007; 276-285.

[78] Xiang Q, Liao B, Xiao L, Lin L, Li S. Discrete-time noise-tolerant Zhang neural network for dynamic matrix pseudoinversion. Soft Computing. 2018. (In press). https://doi.org/10.1007/s005 00-018-3119-8

[79] Xiang Q, Liao B, Xiao L, Jin L. A noise-tolerant Z-type neural network for time-dependent pseudoinverse matrices. Optik-International Journal for Light and Electron Optics. 2018; 165: 16-28. https: //doi.org/10.1016/j.ijleo.2018.03.078

[80] Liao B, Xiang Q. Robustness analyses and optimal sampling gap of recurrent neural network for dynamic matrix pseudoinversion. Journal of Advanced Computational Intelligence And Intelligent Informatics. 2017; 21(5): 778-784. https://doi .org/10.20965/j aciii.2017.p0778

[81] Liao B, Xiang Q. Discrete-time noise-suppressing Zhang neural network for dynamic quadratic programming with application to manipulators. Engineering Letters. 2017; 25(4): 431-437.

[82] Jin L, Liao B, Liu M, Xiao L, Guo D, Yan X. Different-level simultaneous minimization scheme for fault tolerance of redundant manipulator aided with discrete-time recurrent neural network. Frontiers in Neurorobotics. 2017; 11(50): 1-7. https://doi.org/10.3 389/fnbot. 2017.00050

[83] Zhang Y, Ma W, Cai B. From Zhang neural network to Newton iteration for matrix inversion. IEEE Transactions on Circuits and Systems I: Regular Papers. 2009; 56(7): 1405-1415. https: //doi.org/10.1109/TCSI.2008.2007065

[84] Cai B, Zhang Y. Different-level redundancy-resolution and its equivalent relationship analysis for robot manipulators using gradientdescent and Zhang's neural-dynamic methods. IEEE Transactions on Industrial Electronics. 2012; 59(8): 3146-3155. https ://doi .or g/10.1109/TIE. 2011.2106092

[85] Xiao L, Zhang Y. Solving time-varying inverse kinematics problem of wheeled mobile manipulators using Zhang neural network with exponential convergence. Nonlinear Dynamics. 2014; 76(2): 1543-1559. https://doi.org/10.1007/s11071-013-1227-7

[86] Xiao L. A nonlinearly-activated neurodynamic model and its finitetime solution to equality-constrained quadratic optimization with nonstationary coefficients. Applied Soft Computing. 2016; 40: 252259. https://doi.org/10.1016/j.asoc.2015.11.023

[87] Miao P, Shen Y, Huang Y, Wang Y. Solving time-varying quadratic programs based on finite-time Zhang neural networks and their application to robot tracking. Neural Computing and Applications. 2015; 26(3): 693-703. https://doi.org/10.1007/s00521-014-174 4-4

[88] Zhang Y, Ding S, Chen D, Mao M, Zhai K. Zhang-gradient controllers for tracking control of multiple-integrator systems. Journal of Dynamic Systems, Measurement, and Control. 2015; 137(11): 111013. https://doi.org/10.1115/1.4031148

[89] Zhang Y, Zhai K, Chen D, Jin L, Hu C. Challenging simulation practice (failure and success) on implicit tracking control of doubleintegrator system via Zhang-gradient method. Mathematics and Computers in Simulation. 2016; 120: 104-119. https ://doi.org/10 $.1016 / j$.matcom. 2015.07.002

[90] Jin L, Li S, Wang H, Zhang Z. Nonconvex projection activated zeroing neurodynamic models for time-varying matrix pseudoinversion with accelerated finite-time convergence. Applied Soft Computing. 2018; 62: 840-850. https://doi.org/10.1016/j . asoc. 2017. 09.016

[91] Li J, Mao M, Uhlig F, Zhang Y. Z-type neural-dynamics for timevarying nonlinear optimization under a linear equality constraint with robot application. Journal of Computational Applied Mathematics. 
2018; 327: 155-166. https://doi.org/10.1016/j.cam.2017 .06 .017

[92] Jin L, Zhang Y, Qiu B. Neural network-based discrete-time Z-type model of high accuracy in noisy environments for solving dynamic system of linear equations. Neural Computing and Applications. 2018; 29(11): 1217-1232. https://doi.org/10.1007/s00521 $-016-2640-x$

[93] Zhang Y, Ding Y, Qiu B, Zhang Y, Li X. Signum-function array activated ZNN with easier circuit implementation and finite-time convergence for linear systems solving. Information Processing Letters. 2017; 124: 30-34.

[94] Qiu B, Zhang Y, Yang Z. Revisit and compare Ma equivalence and Zhang equivalence of minimum velocity norm (MVN) type. Advanced Robotics. 2016; 30(6): 416-430. https://doi.org/10.1 080/01691864.2015.1120243

[95] Mao M, Li J, Jin L, Li S, Zhang Y. Enhanced discrete-time Zhang neural network for time-variant matrix inversion in the presence of bias noises. Neurocomputing. 2016; 207: 220-230.

[96] Guo D, Zhang Y. ZNN for solving online time-varying linear matrixvector inequality via equality conversion. Applied Mathematics and Computation. 2015; 259: 327-338. https://doi.org/10.1016/ j.amc.2015.02.060

[97] Zhang Y, Qiu B, Jin L, Guo D, Yang Z. Infinitely many Zhang functions resulting in various $\mathrm{ZNN}$ models for time-varying matrix inversion with link to Drazin inverse. Information Processing Letters. 2015; 115(9): 703-706.

[98] Zhang Y, Jin L, Guo D, Yin Y, Chou Y. Taylor-type 1-step-ahead numerical differentiation rule for first-order derivative approximation and ZNN discretization. Journal of Computational Applied Mathematics. 2015; 273: 29-40.

[99] Guo D, Zhang Y. Li-function activated ZNN with finite-time convergence applied to redundant-manipulator kinematic control via time-varying Jacobian matrix pseudoinversion. Applied Soft Computing. 2014; 24: 158-168. https://doi.org/10.1016/j.asoc .2014 .06 .045

[100] Guo D, Zhang Y. Zhang neural network for online solution of timevarying linear matrix inequality aided with an equality conversion. IEEE Transactions on Neural Networks and Learning Systems. 2014; 25(2): 370-382. PMid:24807035. https ://doi .org/10.1109/TN NLS. 2013. 2275011

[101] Xiao L, Zhang Y. Different Zhang functions resulting in different ZNN models demonstrated via time-varying linear matrix-vector inequalities solving. Neurocomputing. 2013; 121: 140-149. https: //doi.org/10.1016/j.neucom. 2013.04.041

[102] Zhang Y, Mu B, Zheng H. Link between and comparison and combination of Zhang neural network and Quasi-Newton BFGS method for time-varying quadratic minimization. IEEE Transactions on Cybernetics. 2013; 43(2): 490-503. PMid:22929435. https: //doi.org/10.1109/TSMCB.2012.2210038

[103] Zhang Y, Yang Y, Cai B, Guo D. Zhang neural network and its application to Newton iteration for matrix square root estimation. Neural Computing and Applications. 2012; 21(3): 453-460. https://doi.org/10.1007/s00521-010-0445-x

[104] Guo D, Yi C, Zhang Y. Zhang neural network versus gradient-based neural network for time-varying linear matrix equation solving. Neurocomputing. 2011; 74(17): 3708-3712. https://doi.org/10.1 016/j.neucom. 2011.05.021

[105] Jin L, Zhang Y, Li S, Zhang Y. Noise-tolerant ZNN models for solving time-varying zero-finding problems: a control-theoretic approach. IEEE Transactions on Automatic Control. 2017; 62(2): 992-997. https://doi.org/10.1109/TAC.2016.2566880
[106] Li Z, Zhang Y. Improved Zhang neural network model and its solution of time-varying generalized linear matrix equations. Expert Systems with Applications. 2010; 37(10): 7213-7218. https : //doi.org/10.1016/j.eswa.2010.04.007

[107] Zhang Y. Revisit the analog computer and gradient-based neural system for matrix inversion. In Proceedings of International Symposium on Intelligent Control. 2005; 1411-1416.

[108] Zhang Y, Chen K, Ma W, Li XD. MATLAB simulation of gradientbased neural network for online matrix inversion. In Proceedings of International Conference on Intelligent Computing. 2007; 98-109.

[109] Chen K, Zhang L, Zhang Y. Cyclic motion generation of multi-link planar robot performing square end-effector trajectory analyzed via gradient-descent and Zhang et al's neural-dynamic methods. In Proceedings of International Symposium on Systems and Control. 2008; $1-6$.

[110] Yi C, Chen Y, Lu Z. Improved gradient-based neural networks for online solution of Lyapunov matrix equation. Information Processing Letters. 2011; 111(16): 780-786. https ://doi.org/10.1016/j. ipl.2011.05.010

[111] Zhang Y, Yang Y, Ruan G. Performance analysis of gradient neural network exploited for online time-varying quadratic minimization and equality-constrained quadratic programming. Neurocomputing. 2011; 74(10): 1710-1719. https://doi.org/10.1016/j.neuc om.2011.02.007

[112] Zhang Y, Chen D, Guo D, Liao B, Wang Y. On exponential convergence of nonlinear gradient dynamics system with application to square root finding. Nonlinear Dynamics. 2015; 79(2): 983-1003. https://doi.org/10.1007/s11071-014-1716-3

[113] Xiao L, Lu R. A fully complex-valued gradient neural network for rapidly computing complex-valued linear matrix equations. Chinese Journal of Electronics. 2017; 26(6): 1194-1197.

[114] Zhang Y, Shi Y, Chen K, Wang C. Global exponential convergence and stability of gradient-based neural network for online matrix inversion. Applied Mathematics and Computation. 2009; 215(3): 13011306.

[115] Zhang Z, Zhang Y. Acceleration-level cyclic-motion generation of constrained redundant robots tracking different paths. IEEE Transactions on Systems, Man, and Cybernetics, Part B: Cybernetics. 2012; 42(4): 1257-1269. PMid:22481829. https://doi.org/10.1109/ TSMCB . 2012.2189003

[116] Zhang Z, Zhang Y. Design and experimentation of accelerationlevel drift-free scheme aided by two recurrent neural networks. IET Control Theory and Applications. 2013; 7(1): 25-42. https: //doi.org/10.1049/iet-cta.2011.0573

[117] Li S, Li Y. Nonlinearly activated neural network for solving timevarying complex Sylvester equation. IEEE Transactions on Cybernetics. 2014; 44(8): 1397-1407. PMid:24184789. https ://doi.org/ 10.1109/ТСYB. 2013.2285166

[118] Chen Y, Yi C, Qiao D. Improved neural solution for the Lyapunov matrix equation based on gradient search. Information Processing Letters. 2013; 113(22-24): 876-881.

[119] Li S, Liu B, Li Y. Selective positive Cnegative feedback produces the winner-take-all competition in recurrent neural networks. IEEE transactions on Neural Networks and Learning Systems. 2013; 24(2): 301309. PMid:24808283. https://doi .org/10.1109/TNNLS. 2012 .2230451

[120] Jin L, Li S, Liao B, Zhang Z. Zeroing neural networks: a survey. Neurocomputing. 2017; 267: 597-604. https://doi.org/10.101 6/j.neucom.2017.06.030

[121] Wilson AD, Schultz JA, Ansari AR, Murphey TD. Dynamic task execution using active parameter identification with the BAXTER 
research robot. IEEE Transactions on Automation Science and Engineering. 2017; 14(1): 391-397. https ://doi.org/10.1109/TA SE. 2016. 2594147

[122] Jin L, Li S, La HM, Luo X. Manipulability optimization of redundant manipulators using dynamic neural networks. IEEE Transactions on Industrial Electronics. 2017; 64(6): 4710-4720.

[123] Xiao L, Zhang Y. Dynamic design, numerical solution and effective verification of acceleration-level obstacle-avoidance scheme for robot manipulators. International Journal of Systems Science. 2016; 47(4): 932-945. https://doi.org/10.1080/00207721.2014.909971

[124] Guo D, Zhang Y. Simulation and experimental verification of weighted velocity and acceleration minimization for robotic redundancy resolution. IEEE Transactions on Automation Science and Engineering. 2014; 11(4): 1203-1217. https ://doi .org/10.110 9/TASE. 2014. 2346490

[125] Guo D, Zhang Y. Acceleration-level inequality-based MAN scheme for obstacle avoidance of redundant robot manipulators. IEEE Transactions on Industrial Electronics. 2014; 61(12): 6903-6914. https : //doi.org/10.1109/TIE.2014.2331036

[126] Zhang Y, Guo D, Ma S. Different-level simultaneous minimization of joint-velocity and joint-torque for redundant robot manipulators. Journal of Intelligent and Robotic Systems. 2013; 72(3-4): 301-323.

[127] Li K, Zhang Y. Design and implementation of a zero-initial-velocity self-motion scheme on a six-DOF planar robot manipulator. Industrial Robot. 2012; 39(4): 401-411. https://doi.org/10.1108/ 01439911211227980

[128] Mohammed AM, Li S. Dynamic neural networks for kinematic redundancy resolution of parallel stewart platforms. IEEE Transactions on Cybernetics. 2016; 46(7): 1538-1550. PMid:26219101. https://doi.org/10.1109/TCYB. 2015.2451213

[129] Zhang Y, He L, Li S, Chen D, Ding Y. Zeroing dynamics based motion control scheme for parallel manipulators. Electronics Letters 2016; 53(2): 74-75. https ://doi .org/10.1049/el . 2016.2307

[130] Mohammed AM, Li S, Jin L. Simultaneous learning and control of parallel Stewart platforms with unknown parameters. Neurocomputing. 2017; 266: 114-122. https://doi.org/10.1016/j.neucom .2017 .05 .026

[131] Zhang Z, Yan Z, Fu T. Varying-parameter RNN activated by finitetime functions for solving joint-drift problems of redundant robot manipulators. IEEE Transactions on Industrial Informatics. 2018. (In press). https://doi.org/10.1109/TII.2018.2812757

[132] Zhang Y, Li W, Liao B, Guo D, Peng C. Analysis and verification of repetitive motion planning and feedback control for omnidirectional mobile manipulator robotic systems. Journal of Intelligent and Robotic Systems. 2014; 75(3-4): 393-411.

[133] Jin L, Li S, Xiao L, Lu R, Liao B. Cooperative motion generation in a distributed network of redundant robot manipulators with noises. IEEE Transactions on Systems, Man, and Cybernetics: Systems. 2017. (In press). https ://doi.org/10.1109/TSMC. 2017.2693 400

[134] Zhang Y, Li S. Predictive suboptimal consensus of multiagent systems with nonlinear dynamics. IEEE Transactions on Systems, Man, and Cybernetics: Systems. 2017; 47(7): 1701-1711. https: //doi.org/10.1109/TSMC. 2017.2668440

[135] Zhang Z, Zheng L, Guo Q. A varying-parameter convergent neural dynamic controller of multi-rotor uavs for tracking time-varying tasks. IEEE Transactions on Vehicular Technology. 2018. (In press). https://doi.org/10.1109/TVT.2018. 2802909

[136] Ma W, Cheng H, Li Z, Qiu J. Time-varying stiffness tracking control of knee exoskeleton. In Proceedings of International Conference on Robotics and Biomimetics. 2017; 1665-1669.
[137] Jin L, Li S. Nonconvex function activated zeroing neural network models for dynamic quadratic programming subject to equality and inequality constraints. Neurocomputing. 2017; 267: 107-113. https://doi.org/10.1016/j.neucom. 2017.05.017

[138] Liu YJ, Chen CLP, Wen GX, Tong S. Adaptive neural output feedback tracking control for a class of uncertain discrete-time nonlinear systems. IEEE Transactions on Neural Networks. 2011; 22(7): 1162-1167. PMid:21659019. https ://doi.org/10.1109/ TNN . 2011.2146788

[139] Zhang Y, Chen D, Jin L, Zhang Y, Yin Y. GD-aided IOL (input Coutput linearisation) controller for handling affine-form nonlinear system with loose condition on relative degree. International Journal of Control. 2016; 89(4): 757-769. https://doi.org/10.1080/ 00207179.2015 .1099075

[140] Zhang Y, Li J, Li S, Chen D, He L. Optimal zeroing dynamics with applications to control of serial and parallel manipulators. Optimal Control Applications and Methods. 2018. (In press). https://doi.org/10.1002/oca. 2417

[141] Zhang Z, Li Z, Zhang Y, Luo Y, Li Y. Neural-dynamic-methodbased dual-arm CMG scheme with time-varying constraints applied to humanoid robots. IEEE Transactions on Neural Networks and Learning Systems. 2015; 26(12): 3251-3262. PMid:26340789. https://doi .org/10.1109/TNNLS. 2015.2469147

[142] Zhang Z, Beck A, Magnenat-Thalmann N. Human-like behavior generation based on head-arms model for robot tracking external targets and body parts. IEEE Transactions on Cybernetics. 2015; 45(8): 1390-1400. PMid:25252290. https ://doi.org/10.1109/ TCYB. 2014. 2351416

[143] Li S, Zhang Y, Jin L. Kinematic control of redundant manipulators using neural networks. IEEE Transactions on Neural Networks and Learning Systems. 2017; 28(10): 2243-2254. PMid:27352398. https://doi.org/10.1109/TNNLS. 2016.2574363

[144] Zhang Z, Zheng L, Yu J, et al. Three recurrent neural networks and three numerical methods for solving a repetitive motion planning scheme of redundant robot manipulators. IEEE/ASME Transactions on Mechatronics. 2017; 22(3): 1423-1434. https ://doi .org/10 .1109/TMECH. 2017.2683561

[145] Li S, Wang H, Rafique MU. A novel recurrent neural network for manipulator control with improved noise tolerance. IEEE Transactions on Neural Networks and Learning Systems. 2017; 29(5): 1908-1918. PMid:28422689. https ://doi.org/10.1109/TNNLS. 2017.267 2989

[146] Zhang Z, Yan Z, Fu T. Varying-parameter RNN activated by finitetime functions for solving joint-drift problems of redundant robot manipulators. IEEE Transactions on Industrial Informatics. 2018. (In press). https : //doi .org/10.1109/TII . 2018.2812757

[147] Jin L, Li S, Hu B. RNN models for dynamic matrix inversion: a control-theoretical perspective. IEEE Transactions on Industrial Informatics. 2018; 14(1): 189-199. https ://doi.org/10.1109/TI I. 2017.2717079

[148] Li W. A recurrent neural network with explicitly definable convergence time for solving time-variant linear matrix equations. IEEE Transactions on Industrial Informatics. 2018. (In press). https : //doi.org/10.1109/TII.2018.2817203

[149] Zhang Y, Chen S, Li S, Zhang Z. Adaptive projection neural network for kinematic control of redundant manipulators with unknown physical parameters. IEEE Transactions on Industrial Electronics. 2018; 65(6): 4909-4920. https ://doi.org/10.1109/TIE.2017.277 4720

[150] Zhang Y, Li S, Gui J, Luo X. Velocity-level control with compliance to acceleration-level constraints: a novel scheme for manipulator redundancy resolution. IEEE Transactions on Industrial Informatics. 
2018; 14(3): 921-930. https://doi.org/10.1109/TII.2017.2 737363

[151] Wang J, Hu Q, Jiang D. A Lagrangian network for kinematic control of redundant manipulators. IEEE Transactions on Neural Networks. 1999; 10(5): 1123-1132. PMid:18252613. https://doi.org/10 $.1109 / 72.788651$

[152] Zhang Y, Li Z, Fan Z, Wang G. Matrix-inverse primal neural network with application to robotics. Dynamics of Continuous, Discrete and Impulsive Systems, Series B. 2007; 14: 400-407.

[153] Chen D, Zhang Y. Minimum jerk norm scheme applied to obstacle avoidance of redundant robot arm with jerk bounded and feedback control. IET Control Theory and Applications. 2016; 10(15): 18961903. https://doi.org/10.1049/iet-cta.2016.0220

[154] Chen D, Zhang Y. Jerk-level synchronous repetitive motion scheme with gradient-type and zeroing-type dynamics algorithms applied to dual-arm redundant robot system control. International Journal of Systems Science. 2017; 48(13): 2713-2727. https ://doi .org/10 $.1080 / 00207721.2017 .1363310$

[155] Zhang Y, Yan X, Zhang Y, Chen D, Tan H. Optimal parameter value of Zhang equivalence for MVN redundancy resolution at velocity and acceleration levels. In Proceedings of International Conference on Natural Computation, Fuzzy Systems and Knowledge Discovery. 2016; 593-597.

[156] Zhang Y, Yang M, Chen D, Li W, Guo D, Yan X. Proposing, QPunification and verification of DLSM based MKE-IIWT scheme for redundant robot manipulators. In Proceedings of Information Technology and Mechatronics Engineering Conference. 2017; 242-248.

[157] Zhang Y, Yan X, Chen D, Guo D, Li W. QP-based refined manipulability-maximizing scheme for coordinated motion planning and control of physically constrained wheeled mobile redundant manipulators. Nonlinear Dynamics. 2016; 85(1): 245-261. https ://doi.org/10.1007/s11071-016-2681-9

[158] Zhang Y, Xiao L, Xiao Z, Mao M. Zeroing Dynamics, Gradient Dynamics, and Newton Iterations. Florida: CRC Press; 2015.

[159] Chen D, Zhang Y, Li S. Zeroing neural-dynamics approach and its robust and rapid solution for parallel robot manipulators against superposition of multiple disturbances. Neurocomputing. 2018; 275 : 845-858. https://doi.org/10.1016/j.neucom.2017.09.032

[160] Zhang Y, Zhang Y, Chen D, Xiao Z, Yan X. From Davidenko method to Zhang dynamics for nonlinear equation systems solving. IEEE Transactions on Systems, Man, and Cybernetics: Systems. 2017; 47(11): 2817-2830. https://doi.org/10.1109/TSMC. 2016.25 23917

[161] Zhang Y, Zhang Y, Chen D, Xiao Z, Yan X. Division by zero, pseudodivision by zero, Zhang dynamics method and Zhang-gradient method about control singularity conquering. International Journal of Systems Science. 2017; 48(1): 1-12. https://doi.org/10.1 080/00207721.2016.1139761

[162] Xiao L, Zhang Y. From different zhang functions to various ZNN models accelerated to finite-time convergence for time-varying linear matrix equation. Neural Processing Letters. 2014; 39(3): 309-326. https://doi.org/10.1007/s11063-013-9306-9

[163] Jin L, Zhang Y, Li S, Zhang Y. Modified ZNN for time-varying quadratic programming with inherent tolerance to noises and its application to kinematic redundancy resolution of robot manipulators. IEEE Transactions on Industrial Electronics. 2016; 63(11): 69786988. https://doi.org/10.1109/TIE.2016.2590379

[164] Jin L, Zhang Y, Li S. Integration-enhanced Zhang neural network for real-time-varying matrix inversion in the presence of various kinds of noises. IEEE Transactions on Neural Networks and Learning Systems. 2016; 27(12): 2615-2627. PMid:26625426. https : //doi.org/10.1109/TNNLS.2015.2497715

[165] Liao B, Zhang Y, Jin L. Taylor $O\left(h^{3}\right)$ discretization of ZNN models for dynamic equality-constrained quadratic programming with application to manipulators. IEEE Transactions on Neural Networks and Learning Systems. 2016; 27(2): 225-237. PMid:26058059. https://doi.org/10.1109/TNNLS. 2015.2435014

[166] Chen D, Zhang Y. Robust zeroing neural-dynamics and its timevarying disturbances suppression model applied to mobile robot manipulators. IEEE Transactions on Neural Networks and Learning Systems. 2017. (In press). https ://doi .org/10.1109/TNNL S.2017.2764529

[167] Chen D, Zhang Y, Li S. Tracking control of robot manipulators with unknown models: a Jacobian-matrix-adaption method. IEEE Transactions on Industrial Informatics. 2018; 14(7): 3044-3053. https://doi.org/10.1109/TII.2017.2766455

[168] Li Z, Yin Z. Zhang dynamics based tracking control of knee exoskeleton with time-dependent inertial and viscous parameters. International Journal of Control, Automation and Systems. 2018; 16(2): 904-911. https://doi.org/10.1007/s12555-017-0011-8

[169] Chen K, Zhang L, Zhang Y. Cyclic motion generation of multi-link planar robot performing square end-effector trajectory analyzed via gradient-descent and Zhang et al's neural-dynamic methods. In Proceedings of International Symposium on Systems and Control in Aerospace and Astronautics. 2008; 1-6.

[170] Zhang Y, Huang H, Yan X, Tan H, Wu S. Inverse-free solution to inverse kinematics of two-wheeled mobile robot system using gradient dynamics method. In Proceedings of International Conference on Systems and Informatics. 2016; 126-132. 